\title{
Re-disciplining Academic Careers? Interdisciplinary Practice and Career Development in a Swedish Environmental Sciences Research Center
}

\author{
Ruth Müller $^{1} \cdot$ Wolfgang Kaltenbrunner ${ }^{2}$ (D)
}

Published online: 30 April 2019

(C) The Author(s) 2019

\begin{abstract}
Interdisciplinarity is often framed as crucial for addressing the complex problems of contemporary society and for achieving new levels of innovation. But while science policy and institutions have provided a variety of incentives for stimulating interdisciplinary work throughout Europe, there is also growing evidence that some aspects of the academic system do not necessarily reward interdisciplinary work. In this study, we explore how mid-career researchers in an environmental science research center in Sweden relate to and handle the distinct forms of uncertainty that arise from conflicting institutional and policy impulses. Our material suggests that interdisciplinary academics are often confronted with and at times themselves operate with a surprisingly dichotomous, value-laden view of their research practice. Disciplinarity is primarily associated with the ideals of scientific rigor, while interdisciplinarity becomes conflated with application-oriented work and a lack of 'theory.' We also draw attention to the underlying practical dynamics that reproduce this tension and entangle it with the very process of academic socialization. Specifically, we analyze the ambivalent consequences of the various work-arounds that researchers rely on to carve out opportunities for ongoing interdisciplinary research within heterogeneous funding landscapes. These tactics turn out to be undermined by the overriding normative power of formal career incentives at universities, which continue to emphasize the ideals of the individual high-performing academic who publishes in disciplinary journals and attracts the most selective grants. Under such
\end{abstract}

Wolfgang Kaltenbrunner

w.kaltenbrunner@cwts.leidenuniv.nl

Ruth Müller

ruth.mueller@tum.de

1 Munich Center for Technology in Society \& School of Management, Technical University of Munich, Augustenstr. 44-46, 80333 Munich, Germany

2 Centre for Science and Technology Studies, Leiden University, P.O. Box 905, 2300 AX Leiden, The Netherlands 
circumstances, the work-arounds themselves become an insidious mechanism that allows researchers to stay in academia but systematically marginalizes their voices and epistemic ambitions in the process.

Keywords Interdisciplinarity $\cdot$ Science funding $\cdot$ Academic career $\cdot$ Sweden

\section{Introduction}

Over the last two decades, fostering interdisciplinarity in research and innovation has been a central goal of European science policy. Interdisciplinarity is often framed as crucial for addressing the complex problems of contemporary society and for achieving new levels of innovation. The launch of the latest European Union framework program Horizon 2020, for example, has been accompanied by frequent programmatic declarations by policy actors stating that interdisciplinary research would be a "key to future scientific breakthroughs" (Science Europe 2012: 3) and a crucial instrument for addressing 'grand challenges' such as climate change or new energy sources (Allmendinger 2015). Similarly, national science policy across Europe regularly emphasizes the value of interdisciplinarity for both basic and applied research. Sweden, for example, the national context in which the case study we present in this paper is set, regularly emphasizes the value of interdisciplinarity for science and society, which is, for example, also expressed in its diversified landscape of funding bodies that often pursue specific 'sectoral' agendas to address pressing societal and ecological problems (Jacob 2015; Håkansta \& Jacob 2016). This push for interdisciplinarity has led, among other things, to the emergence of numerous interdisciplinary training programs on the Master and $\mathrm{PhD}$ level across Europe that aim to prepare a new generation of scientists for the challenges and opportunities of interdisciplinary work (Lindvig \& Hillersdal 2019). It has further led to the establishment of interdisciplinary research centers that aim to offer dedicated environments for facilitating interdisciplinary work practices among scientists with a diversity of backgrounds. Yet, while this movement towards the establishment of interdisciplinary institutional elements is ongoing, there is also growing evidence that some aspects of the academic system do not necessarily reward interdisciplinary work. A number of studies, for example, suggest that the contemporary evaluation and funding system tends to privilege disciplinary work and might promote the careers of disciplinary scholars over those of interdisciplinary researchers (Hamann 2016; Rafols et al. 2012; Whitley 2010).

In this article, we are interested in exploring how researchers who work in a dedicated interdisciplinary environment, in our case an environmental science research center in Sweden, relate to and handle the distinct forms of uncertainty that arise from these conflicting institutional and policy impulses. We specifically focus on mid-career researchers (postdocs, assistant professors), as prior studies have shown that this group is both particularly vulnerable to and aware of the norms and values of academic reward and career systems (Fochler et al. 2016; Müller 2012, 2014a, b). We explore how researchers at this specific career stage in an academic environment 
that is explicitly dedicated to interdisciplinary work experience and relate to questions of inter/disciplinarity as they aim to build their careers as researchers. Our research shows that bound up in this discussion of inter/disciplinarity are also questions about the place of social relevance and political engagement in science and about its value for academic career development. Negotiations about inter/disciplinarity in this sense are also, as we shall see, contestations about what constitutes valuable science in the 21 st century and if there is room for more than one such definition.

\section{Literature Review: Three Approaches to Studying Interdisciplinary Research}

Existing studies of interdisciplinarity to date can roughly be distinguished into three main strands. A first strand is primarily concerned with a descriptive typology of interdisciplinary research. A variety of authors have, for example, proposed possibilities for differentiating research in terms of inter-, trans-, and multidisciplinarity (e.g., Boden 1999; Klein 2000, 2010; Lattuca 2001). According to a currently dominant usage (Klein 2000), the term multidisciplinarity, for example, denotes a situation in which a single research topic is investigated from a variety of disciplines. However, the disciplinary perspectives involved are not actually integrated but are mobilized in a parallel or sequential fashion. Interdisciplinarity by contrast would be a research practice that integrates epistemic resources from different fields in the very process of knowledge production, thus resulting either in new hybrid research approaches, or in stable bridges between existing disciplines. Finally, a common usage of the term transdisciplinarity is to denote the joint production of knowledge through academic and non-academic actors, for example, in industrial/private sectors. The basis of such taxonomic distinctions is thus a somewhat idealized conceptualization of disciplines and academic science as entities with sharply defined edges, which can relate to each other in a range of specific ways.

A second strand of literature has empirically studied the challenges of interdisciplinary work for day-to-day research practices and specifically for career development (Hackett \& Rhoten 2009; Pfirman \& Martin 2010; Choucri et al. 2006). For example, it has been stated that interdisciplinary researchers often work on a range of topics rather than specialize on one specific issue, which has been found to negatively impact the frequency of publication in the long run (Porter et al. 2007; Leahey 2007; Spanner 2001). As interdisciplinary work is also often arranged in collaborative structures, other studies have examined the higher-than-usual transaction costs inherent to interdisciplinary work that occur, for example, because researchers need extra time to achieve a baseline conceptual and methodological consensus in order to collaborate (Pfirman \& Martin 2010). Finally, a number of studies have examined the relative invisibility of interdisciplinary publishing as compared to disciplinary publications (Rhoten \& Pfirman 2007; Campbell 2005). Apart from highly visible journals open to multiple disciplines, such as Nature and Science, dedicated interdisciplinary journals are fewer in number and have smaller audiences compared to disciplinary outlets. Beyond reaching a limited audience, this can also have a 
secondary effect on the career development of interdisciplinary researchers as, for example, tenure committees often look for publications in established high impact journals, and these tend to be disciplinary in nature (National Academies 2005; Boix Mansilla 2006; Boix Mansilla et al. 2016). Much of this second strand of literature is primarily empirical in its orientation, with relatively little in the way of theoretical framing. Moreover, this literature is distinct from the first strand in that it does not usually interrogate or aim to define interdisciplinary research as such. As a result, the notion of interdisciplinarity tends to remain somewhat unspecific, and it is often unclear in which particular contexts interdisciplinary work is easier or more difficult to perform. For example, do the conditions provided by different national science systems play a role? Are all forms for interdisciplinary research equally affected?

Yet a third body of literature adopts a more reflexive stance in the sense of relating definitional questions to the specific institutional and policy context in which interdisciplinary work is conducted (e.g., Barry et al. 2008; Strathern 2007; Felt et al. 2013; Felt et al. 2016; Lindvig \& Hillersdal 2019; Weingart \& Stehr 2000). Felt et al. (2016) have studied transdisciplinary research projects in Austria in which researchers are meant to collaborate with non-academic actors to develop and tackle joint research problems. However, their study abstains from providing any a priori analytical definition of such collaborative formats, and instead focuses on how their actors rhetorically switch between different models of interaction, depending on situational needs. In some situations, it is more useful for the involved actors to promote a more traditional disciplinary research model where academic expertise is posited as more authoritative than the expertise of societal stakeholders. In other cases - for example, when interacting with funders - academics may decide that it makes more sense to stress the equality of different forms of knowledge.

A similar picture arises from a recent study by Lindvig \& Hillersdal (2019), in which they report findings from their ethnographic field work in a large interdisciplinary research program in Denmark. They suggest that the researchers and administrators who initiated the program used the notion of interdisciplinarity primarily in a symbolic way to attract funding and political legitimacy for their institution. However, unclear definitions regarding the intended program outcomes and how they would be evaluated, combined with a lack of concrete measures to foster interdisciplinary collaboration on a day-to-day basis, quickly made program participants fall back on more established monodisciplinary routines.

Although appeals to interdisciplinarity thus can play an important role in mobilizing funding and legitimacy vis-à-vis science policy, this does not mean that it can be reduced to a mere label researchers opportunistically attach to their activities when it makes sense. Barry et al. (2008) have called for more empirical attention to how specific forms of interdisciplinarity emerge from a situated interaction between institutional and funding conditions and epistemic aspects. For example, when approaches from the social sciences are integrated into other disciplinary contexts, they are often expected to serve a substantive instrumental function. A case in point would be the use of ethnographic methods to facilitate user-centered design in human-computer interaction research. Strategic considerations of the actors involved here often play a role - such as researchers in marginalized fields looking for new sources of funding and legitimacy - but in ways that may well lead to 
the consolidation of specific interdisciplinary approaches in the shape of dedicated research institutions or research agendas.

Lastly, Marilyn Strathern $(2004,2007)$ grounds her reflections on interdisciplinarity in a broader inspection of recent changes in the institutional organization and funding structure of British (and other) universities. Newly proactive science policymakers, she argues, have often regarded the disciplinary structure of academic work as an array of artificial epistemic holding patterns that restrict intellectual innovation and thus also the efficient use of public funds. According to Strathern, a dominant assumption behind funding programs for interdisciplinary work in the UK and other European countries is that they will effectuate a broadening of disciplinary quality standards and thus subject research to alternative forms of accountability (e.g., relevance to industrial or other societal stakeholders).

We find this third strand of literature particularly enriching because it makes visible that the specific nature and the intensity of the challenges researchers often encounter when conducting interdisciplinary work - specifically with regard to career development as the empirical studies we discussed before show - can be a result of interactions between different contextual factors, such as novel and strategic funding incentives and the specific academic landscapes in which they unfold.

\section{Anticipatory Uncertainty and Interdisciplinary Research}

With our own analysis we want to contribute to this last strand of research. Specifically, we will provide a detailed empirical case study that relates the level of dayto-day knowledge production in an interdisciplinary Swedish research center at the intersection of the natural and social sciences to its broader institutional and policy environment. In so doing, we take inspiration from the concept of anticipatory uncertainty as proposed by Fochler and Sigl (2018).

At the heart of Fochler and Sigl's analysis is the attempt to understand how epistemic dynamics in research are intertwined with the institutional organization of science in terms of career structures and funding. STS literature has a long tradition of studying how researchers deal with the inherent uncertainties involved in scientific discovery, e.g., whether particular research practices will produce reliable results or correspond to the natural processes studied (Knorr-Cetina 1981; Star 1985). Fochler and Sigl argue that changes in the social organization of research since the 1980s, including a projectification of research, increasing competition for funding, and formalized ways of assessing academic performance have led to a problematic entanglement between such epistemic uncertainties and social uncertainties related to careers and funding. Researchers experience pressures to constantly monitor their performance and account for their successes and failures in the rather short intervals that depend not on the 'eigentime' of epistemic processes but on the durations of research projects and employment contracts. To avoid that disappointing research results become an immediate threat for the careers of individual scientists, researchers need to systematically limit epistemic risk, e.g., by avoiding overly ambitious research projects or balancing them with activities that are more likely to yield predictable but less exciting results (cf. also Müller 2014a). Fochler and Sigl argue that 
the perpetual need to organize all research activities in light of their anticipated formal productivity (in terms of publications, deliverables) amounts to a new form of de-facto governance of scientific work. One of its main effects is to hamper the ability of scientists to productively embrace the intellectual and practical challenges that are often involved in intellectually interesting and/or societally relevant research.

In this paper, we will analyze how different impulses by funders, peers, institutions and policy actors in Sweden have effectively created contradictory incentives with regard to the value and import of interdisciplinary research. Interdisciplinary work has, on the one hand, been encouraged as it is considered a source of highly socially relevant research by Swedish policymakers. A particular focus of funding bodies has been research on environmental issues at the intersection of the natural and social sciences. These incentives are part of transformations in the science policy landscape at European and national levels that aim to stimulate types of academic knowledge production that focus on problem-solving and involve stakeholders from industry, policy, and civil society (Nowotny et al. 2001; Funtowicz \& Ravetz 1993; Fisher et al. 2001; Wald 2007). At the same time, a strand of parallel reforms has tended to rub against systemic measures that encourage researchers to transcend disciplinary and institutional boundaries. Sweden operates with an increasingly formalized research evaluation system that places a strong emphasis on publications in reputed international journals, as well as success in prestigious grant schemes (Sivertsen 2008; Hammarfelt \& de Rijcke 2015; Hicks 2012). Although the exact ways in which such evaluation systems affect research practices in specific fields are still rather poorly understood (Hammarfelt \& de Rijcke 2015), a common effect of formalized research evaluation seems to be institutional stratification. Standards of good research as enacted by classifications of publication venues are often modeled on the judgment of elite researchers who represent orthodox epistemic agendas, thus resulting in a systematic channeling of symbolic and material resources towards well-established disciplinary institutions (Hamann 2016; Rafols et al. 2012; Whitley 2007).

How interdisciplinary research is affected by the interaction between these diverse policy measures is an important but yet understudied problem. With this paper we contribute to filling this gap by exploring questions such as through which strategies do researchers try to reconcile funding opportunities for interdisciplinary, 'societally relevant' work, on the one hand, and the need to accommodate funding and publication practices that are often geared towards disciplinary research, on the other? Further, how do their strategies for managing uncertainties regarding careers, funding, and publication performance affect and shape their ability to tackle the epistemic and practical uncertainties involved in interdisciplinary research?

\section{Material and Methods}

The material for this article was collected at an interdisciplinary research center that is specialized in research on contemporary environmental challenges. Founded in the mid-1990s, the center is situated at a Nordic university with a significant tradition in interdisciplinary research. It hosts about 20-30 researchers with educational 
backgrounds in both the natural and social sciences, with some holding degrees in both areas or interdisciplinary degrees. We have used a combination of methods to collect data. This includes several multi-day research visits in 2014 as well as participant observation at meetings at the center. The core of our empirical work consists of 14 semi-structured interviews (2-3 hrs) with postdocs, assistant professors, and key senior researchers. Conducted according to the reflexive peer-to-peer method (Fochler et al. 2016; Müller 2014b), the interviews were approached as conversational situations among equals, where the interviewer (Müller) encouraged the interview partners to reflect on otherwise taken-for-granted principles and norms that underpin working and living in an interdisciplinary research center. Specifically, the first part of topic guide was designed to encourage researchers to recapitulate their scientific biographies, i.e., their original motivation for academic work, their concrete career experiences, and their future ambitions. The second part addressed the current research practices of our interviewees, focusing in particular on the epistemic and social organization of their work. In a final set of questions, we focused on institutional and policy factors that posed obstacles or enabled their work. Here, the interviewer often followed up on themes and concrete situations that had emerged throughout the conversation. To analyze the data, we adopted a grounded-theorybased approach involving multiple rounds of open and focused coding (Charmaz 2006; Strauss \& Corbin 1998).

\section{Empirical Analysis}

Our empirical analysis is structured as follows: First, we take a brief contextualizing look at the historical circumstances under which the research center in question was founded. We draw particular attention to pragmatic funding considerations and the development of an agenda for interdisciplinary institution-building. Second, we look at practices of interdisciplinary work at the center. We discuss factors that, according to our respondents, have helped to foster a productive climate for interdisciplinary research, and then analyze two typical occasions where disciplinary ideas of 'proper' research have rubbed against the interdisciplinary practices cultivated at the center. In a third step, we analyze how researchers try to reconcile such competing frames of reference and investigate the strategies researchers at the center apply in order for their work to 'pass' as either disciplinary or interdisciplinary, depending on situational needs. Yet, in a final step, we critically examine how sustainable these practices are for mid-career researchers, particularly with regard to more long-term career development. A central conceptual feature of our empirical narrative thus is to interweave two analytical layers: That of the origins and gradual consolidation of the interdisciplinary research center, on the one hand; and that of individual members and how they maneuver career and funding structures of the Swedish academic system, on the other. This combination, we suggest, is a crucial for understanding interdisciplinary work as a set of practices with a distinct historical and institutional context. It is on the basis of such a detailed contextual understanding that we can 
better understand both the challenges interdisciplinary researchers encounter, as well as the strategies they may develop to tackle them.

\section{Interdisciplinary Research and Institution Building}

The origins of the interdisciplinary research center that was our field site can be traced back to the 1980 s, when its host university established a variety of small issue-focused research units outside its regular departments. Vested with a handful of professorships and a doctoral program, the units focused on broadly conceived interdisciplinary topics of high social relevance. One of these units specifically sought to encourage graduate students from the social and the natural sciences to carry out interdisciplinary research on contemporary environmental issues. The underlying normative assumption - widespread both in policy and academic circles - was that many contemporary ecological challenges pose "wicked problems" (Rittel \& Webber 1973) that cannot be adequately addressed from one single disciplinary perspective but require knowledge and expertise from both natural and social scientific domains (Sarewitz 2010; Felt et al. 2013). The environmental studies unit at that point was funded and administrated jointly by the faculties of science and social science. However, in the mid-1990s, a historical opportunity arose for the affiliated researchers to turn their unit into an actual research center with a more independent institutional foundation. Important preconditions for this move were the governmental decision to expand higher education offerings in the specific region of the university, as well as a new demand by public administrators for practical advice on particular problems of environmental policymaking. In this context, researchers of the environmental studies unit managed to convince the university leadership to support the creation of a dedicated research center. Yet, while they received the goahead, the condition was that they would be able to attract an external grant to provide seed money. The necessary resources were ultimately secured through a grant from a Swedish governmental agency, which had issued a thematically very suitable funding call just shortly before. A founding member of the center recalls:
"There had been some [higher] education in [the region of the university], but in the mid-90s the present government wanted to expand it (...), so rather than forming a university of its own, (...) [the university] decided to have a strong campus here with both more education programs and research. (...) And there the thought of having [this type of environmental] research here then came about, to see how that could happen. It was not prioritized I would say, because the dean of the university didn't put any money on the table, he just said, okay, if you can bring in a major grant, then we can start the center (...) So then we went out and (...) started to plan for a grant from [Swedish governmental agency], which had a call out for [environmental] policy." (Senior researcher)

The possibility to succeed in applying for funds from thematic grant programs like this one also informed the center's general institution-building strategy in the following years. Initially, affiliated researchers aimed to base the reputation of their institution primarily on providing academic advice for policymakers, as well as on a 
strong presence in public debates that played out in the Swedish media. The center was so successful in its engagement activities that it quickly came to be considered a showcase for the university. Yet, after a few years of focusing primarily on engagement with policy actors and the public, the members gradually came to the conclusion that while their public impact was significant, they were not necessarily as influential in academic circles as they would have liked to be. Thus, the leadership felt the need for all researchers at the center to start focusing more on traditional forms of 'output,' too, such as publications in international journals and presentations at academic conferences.

"I think [cultivating a media presence through interviews and opinion pieces] was, I think it was important to establish us, not least the university got the image that we are active and we are an important voice. And I thought it helped us eventually to get collaborations, because people knew about us and colleagues knew about what we were doing (...). But after a while we said, no okay, (...) now we have to focus on getting the peer reviewed papers out, because I realized we were, I thought we were doing more poorly than we should in getting our peer reviewed papers out, we got some, but not to the extent that I think we should have." (Full professor)

This tension between contributing to public debate and policymaking, on the one hand, and scoring well in terms of academic performance indicators, on the other, would become characteristic for much of the work at the center, as would, to a certain degree, the (reluctant) deficit narrative ('social impact is not enough') that is present in this senior researcher's account.

\section{Fostering a Culture of Interdisciplinary Thought}

Although not initially allowed to appoint professors and lecturers of their own, the foundation of the center through the government grant afforded its members a much greater discretion over the use of resources than in the previous arrangement. That made it possible to cultivate dedicated interdisciplinary research agendas and working formats. Certainly, the center's collective vision of interdisciplinarity is best seen as an ideal that members aspired to but arguably did not universally realize in practice. As is well known, interdisciplinary research comes with specific challenges, such as additional work to establish common epistemic ground between collaborators from various backgrounds, as well as the lack of obvious conceptual and methodological templates (Porter et al. 2007; Pfirman \& Martin 2010). Our data indicates that collaborative undertakings at the center continued to struggle with these challenges. For example, collaborators often disagreed about the extent to which they could be expected to immerse themselves in the conceptual frameworks of their colleagues, especially in the context of joint writing projects. Moreover, the center's interdisciplinary ambitions were at times seen in a critical light by doctoral students. One PhD student stated that she enjoyed the intellectual diversity at the center but at the same time missed the 
"sharp tools" or "specific toolbox" she assumed a monodisciplinary framework would have provided for her doctoral research.

While opinions about the success of individual undertakings thus differ, informants agree to a remarkable extent on the organizational and practical aspects that were conducive to interdisciplinary work. Particularly important here seem to have been a conversational culture characterized by "low prestige." By this term a variety of our informants sought to convey a trustful intellectual atmosphere in which one "dare[d] to ask the questions we wouldn't ask in any other environment." This includes not being afraid to admit one's ignorance of particular theoretical concepts, for example, by asking "what is that 'ism' again or what does it mean (...), just tell me again because I can't remember." Confirming the particular importance of trust in interdisciplinary collaboration as observed in previous studies (Boix Mansilla et al. 2016), center members thus appreciate not having "to pretend to know more than you do." Another key ingredient of the specific conversational culture that members deemed so important for interdisciplinary work could perhaps be labeled 'intellectual musing' (our term). This entails the possibility to regularly meet up and engage in non-targeted exchange in a shared physical setting, i.e., a social occasion in which researchers can bring in expertise from different disciplinary angles, but without presupposing a specific practical outcome (cf. Parker \& Hackett 2012). The perceived importance of such informal get-togethers has recently begun to stand out even more, precisely because researchers at the center find that very practice of intellectual musing increasingly difficult to afford. For one, a managerial decision by the university administration mandated that research and administrative meetings should be split across two separate campuses. In practice, many researchers have since begun to attend the research meetings rather selectively, carefully weighing whether the time spent in public transport is worth the effort. Secondly, and more generally, various informants described a gradual change in the way center members managed their working hours. Two founding members we interviewed noted that researchers at the center nowadays were much less willing to engage in non-targeted conversation and sharing a coffee when compared to the early days of the center. This also impacts the general approach to interdisciplinarity, insofar as such time spent together was a crucial element of the intellectual culture at the center.

"So, I want to go and have coffee or I want to just kind of chill a bit, but then there is no one to have coffee with, because they are running and then if I do drag them to coffee - which I think is good - then I feel partly guilty, because they should really have been writing a proposal and I took their fifteen minutes from that. (...) we used to laugh here all the time, that's why I came here, even when I didn't belong here formally. It was really fun, people laughed all... we were people who used to get annoyed at the neighbouring department, because they were always... you know kind of "It's a university, shut your door!", you know, we were sitting here laughing and we still did good research. It is not like we just were fooling around and doing dumb stuff (...)." (Senior researcher) 


\section{Competing Frames of Reference}

This somewhat vague impression that the interdisciplinary practices cultivated at the center are increasingly difficult to keep up can be further interrogated by looking at formal occasions at which the work of center researchers are subjected to quality judgments by external, often disciplinary audiences. For one, when doctoral students submit their theses, review committees are assembled that usually also include disciplinary researchers. A recurring experience has been that doctoral theses were enthusiastically received by interdisciplinary and non-academic audiences with a particular interest in the topic (such as policymakers) but were found to be insufficiently grounded in a disciplinary framework by external $\mathrm{PhD}$ examiners. One such experience in particular - recounted in the following - has become part of the collective memory of the center.

"One concrete example was here, for instance, Sven who I was co-supervisor to, and I think that was quite tough when he had his dissertation [defense]. (...) in many people's eyes it was quite unfair, because the dissertation became sort of stopped with a theoretical framework and then all the sort of good stuff that was in the dissertation was never sort of touched upon during the [defense] (...) but then the sort of interesting thing which shows I think again what we are doing in an interdisciplinary environment as ours might not be appreciated by scientists from individual disciplines (...) for instance, I was in an expert group this autumn $[\ldots]$ on a high governmental level and that's the sort of group that decides on our environmental politics. (...) they referred a lot to what Sven is doing and he has been there now, I think just a week ago, doing some sort of briefing for the politicians and so on. So, I think much of what we are doing is seen on a sort of decision level (...) as something that's extremely important, but from a strict academic level it might be seen as not theoretical enough." (Full professor)

Sven's story is of particular analytical interest. Not only does it relate a 'real story' that has taken place, but it has also ever since been used as a narrative device through which our interview partners spoke about their own struggles with how to orient their work. Senior researchers have taken this particular defense as a sort of prompt to reflect on potential changes in the direction of $\mathrm{PhD}$ supervision, so as to better prepare students for the academic labor market, which is perceived to still operate largely in disciplinary terms. In these types of reflections, speakers again tend to construct a dichotomy where they align interdisciplinarity with the "applied" or the "societally relevant" and oppose it to disciplinary research which they assume has theoretical rigor but remains of interest mainly to academic audiences:

"There have been a few dissertations now where during the defense we have had opponents that have been, for instance, from political science or something with a very disciplinary view and they haven't really considered what has been presented as good science, because it doesn't use the right theoretical framework, it doesn't even in some people minds have a theoretical framework, it is too applied and so on. So, I think the, it has been more stressful now that I 
think many of the supervisors really feel that we have to go back and leave this interdisciplinary environment that we really want to have." (Full professor)

For fellow PhD students, Sven's story has served as a memorable indication that graduate training at the center could pose problems for career development in Swedish universities. The arguments that played out at the defense have been taken to exemplify a structural mismatch between the type of research encouraged by senior members of the center, and what disciplinary elites in other fields consider evidence of scholarly potential. In our interview, Sven himself described the public defense of his thesis primarily as an experience of unpleasant surprise. Reflecting on his graduate and $\mathrm{PhD}$ studies, he admitted to be conflicted about the type of education he had received. While he still saw the exposure to different disciplinary frameworks as inspiring and important, he also felt that he might have been insufficiently prepared for an academic landscape characterized by strong disciplinary conventions. At the time of our interview, he wondered whether it would not make more sense for him to leave the university and take up a job in public administration or with an NGO.

Another occasion where center-specific conventions regularly clash with the expectations of other audiences is when postdocs begin to apply for permanent positions, either at their university or at other institutions. The university that hosts the center operates with a number of tenure requirements. These are largely co-extensive with general Swedish research evaluation modalities, including consideration of raw publication counts in reputed international journals, citation-based metrics like the h-index, as well as researchers' ability to attract grant money (Sivertsen 2008). Below, one young assistant professor recounts her understanding of what she needs to do to make a career at the university:

"Well, first of all publish of course, but don't just publish anywhere, publish in highly ranked international journals. And we have been on the basis of that also been evaluated, so that, no that was the initial criteria, but then it has been qualified as a, also high impact journals and we have to, so we have been evaluated on the basis of our citation indices. And according to a natural science variable, the h-index which was totally unknown to me before, which forces me then to go into the Web of Science and actually check my h-index which is absolutely crazy for a social scientist I think, but that's how they want to do it. So, it was a natural science template very much that informed these criteria. And then we were expected to build research networks and be evaluated on the basis on that. And we should develop a research group, we should of course, now I am forgetting one of the more important ones, ah attract external grants." (Assistant professor)

None of the tenure requirements as informally recounted by our interviewee make any specific statement about the disciplinary orientation of candidates. However, there is a wealth of anecdotal evidence suggesting that current forms of measuring academic impact tend to privilege disciplinary forms of research. Research published in interdisciplinary journals is said to achieve limited and slow reception among disciplinary 'core' audiences, whose judgment plays a very important role in the competition for tenured positions and professorships (Leahey 2007; Rhoten 
\& Pfirman 2007; Rinia et al. 2001). Moreover, while the Swedish research funding system is rather diversified, the individual funding sources are ranked in an implicit hierarchy. According to a widely shared sentiment among our informants - and arguably other Swedish academics - grants by the Swedish research council are considered to be more prestigious than those offered by specific sectorial ministries. As a result, center researchers are often caught between contradictory frames of reference: The specific brand of interdisciplinary research as cultivated in the center over the years, on the one hand, and academic tenure requirements that are tailored to disciplinary funding and publishing practices, on the other.

\section{Maneuvering Different Frames of Reference}

Our interviews point to a number of strategies researchers have learned to rely on to reconcile the above-described tensions. One strategy, documented in a range of previous studies (Hackett 1987; Laudel 2006; Sigl 2015; Fochler \& Sigl 2018), consists of combining resources from diverse funding bodies by pooling grants and universty funds into a shared budget. This collective resource buffer buys researchers a degree of epistemic autonomy, for example, the possibility to pursue lines of inquiry that did not (yet) receive dedicated funding. Researchers at the center similarly draw together grants at the institutional level, thus creating a degree of employment continuity even for members without long-term positions.

A second strategy through which researchers try to reconcile interdisciplinary ambitions with the career norms of their institutional environment is the use of research portfolios (Leisyte 2007; Gläser et al. 2010). It consists in diversifying the center's range of grant applications in such a way as to be able to react to sudden shifts in funding trends. In particular, some center members anticipate that political interest in the environmental policy problems that the center has traditionally focused on will begin to wane in the short or medium term. However, an internal bibliometric report circulated as input for the further development of the center's institutional strategy positively observed that a significant amount of center publications actually tended to stray from the original environmental policy focus towards related topics like water management, participation, and environmental communication. The report concludes that if funds for the original policy theme were to dry up, the center could adapt by shifting towards these already emerging areas.

Yet a third strategy we were able to identify is a specific practice of producing grant applications. It consists in writing research proposals in such a way that they can be tailored to the expectations of both 'basic' and 'applied' funding bodies with some adjustments. In the following, a senior researcher explains how she adapted a successful research proposal originally submitted to the Swedish research council for another grant application with a sectorial research council.

A: “(...) in the VR proposal, we focused on why this is interesting from an international relations point of view. (...) the focus of the [sectorial application] was slightly different. We still address those issues, but focus more on the role of non-state actors in [a specific area of policy-making] (...) so it was not a huge difference [between the two versions], because (...) these three ... 
methodological, theoretical and sort of the more practical [environmental] policy $(. .$.$) focus (. .$.$) was in all three applications but with a slight shift in what$ was in the forefront." (Full professor)

The quote illustrates a practice of adjusting what Peterson (2017) has called the epistemic "depth of field" of research proposals. In optical terminology, depth of field denotes the range of distance in an image that is sufficiently sharp for observers to recognize details. The farther away objects are located from this focus, the more blurry they become. Peterson proposes this photographic metaphor to capture the rhetorical practices through which scientists manage the focal points of their research interests. Epistemic communities generally grow by aligning their work around shared areas of attention, while dismissing questions that lie outside as either too narrow or too broad. However, when addressing readers outside their community, scientists may have to adjust the focal point of their argument if they wish to convince the respective audiences of the relevance of their work. Our respondents leverage this principle through producing essentially modular research proposals. Proposals are built around a conceptual core of an argument whose diverse aspects can be zoomed in on depending on situational needs. Increasing the level of theoretical detail and a deeper embedding into the scholarly literatures provides grounding in disciplinary discourses, while a broader contextualization of the main argument, combined with references to ongoing public debates, increases relevance to policy.

\section{Funding Sources and Social Hierarchies}

The strategies detailed in the previous section can in principle be used by all researchers who find their research agendas more or less ill-aligned with dominant incentives in their funding environment. Inevitably, however, the first two of these strategies can only be used at the institutional level of a research center or department. Their shared principle is to decouple the epistemic logic of specific research projects from the budgetary logic of the whole center, and thus to prevent that any failure in grant applications or otherwise disappointing outcomes of research immediately become career-threatening for individual researchers (cf. Sigl 2015). Another way of putting this is to say that the pooling of funds and the use of research portfolios require at least a degree of institutional solidarity. After all, both strategies are based on redistributing resources from well-performing to more poorly performing researchers, projects and topics.

We found, however, that such redistribution of funds has implications for the social status of the researchers who have to rely on them. As a general rule, the more grant money a researcher is able to bring in, and the less he or she has to rely on others to guarantee employment continuity, the better his or her standing is assumed to be within the center. Consequently, researchers worry that if they come to contribute very little or nothing to the institutional budget, they might find themselves at the very bottom of the informal social hierarchy and might be seen as "slackers" or "free-loaders." But our material also reveals a sophisticated hierarchy among those researchers who actually manage to make substantial contributions to the shared budget. More specifically, our interview partners routinely evoked a dichotomy of 
'ideal types' of researchers who tie the collective perception of individuals to the specific funding sources they manage to tap into.

The first ideal type we encountered is the "politically correct researcher." Although his or her contributions to the shared budget pool may be substantial, they are primarily drawn from sectorial funding bodies, such as the Swedish Research Council for Environment, Agricultural Sciences and Spatial Planning (Formas) or the Swedish International Development Agency (Sida), which adopt a thematic focus and explicitly encourage interdisciplinary work. Formas typically focuses on projects addressing ecological challenges, while Sida is specialized on research for international development. As one senior researcher stated, academics both in- and outside the center commonly suspect that projects funded by the two agencies are not primarily selected because of their intellectual merit, but because of their opportunistic compatibility with (shifting) fashions in the policy discourse: "you are (...) able to attract funding because you are politically correct."

In the common perception of researchers at the center, the charge of political opportunism and the foci of these specific funding sources combine to create a diffusely negative image of "politically correct researchers." It seems that internalizing this negative view and the normative hierarchy of funding sources it implies constitutes an important step in the gradual enculturation of young researchers in Swedish academia. For example, one of our interview partners reported that it took her quite a bit of time to understand why more established colleagues were underwhelmed to learn that someone in the center had attracted a grant from Sida. Outlining an ascending imaginary 'ladder' of funding sources with her hands, she explained: "I didn't even know there were gradings of money, you know, like Sida I guess is down here [gesturing with her hands]. VR is there and Formas is I think slightly better than Sida, because at least you are doing something in Sweden."

By contrast, the second ideal type is what one informant called the "reliable academic," i.e., a researcher whose capacity to bring in funds is characterized by greater versatility of sources compared to the "politically correct" type. Not only do "reliable academics" manage to maintain a particularly high rate of successful grant applications over time, but they are also able to draw funds from the whole spectrum of funding sources. This includes grant-giving bodies that do not combine their funding frameworks with any thematic programming efforts, such as the Swedish research council VR and potentially the European research council. For an interdisciplinary research center, "reliable academics" are valuable in a double sense. Firstly, they can be counted on to help maintain a specific budgetary mix, and secondly, they are useful to demonstrate academic excellence to the university administration and to peers with a more disciplinary research orientation. This becomes particularly explicit in the following quote, where a professor recounts how a successful grant application strengthened her bargaining power vis-à-vis the university leadership.

A: "So, [the dean] emphasized very much the importance that we get funded from [VR] to show that we are credible in the scientific fields. So, for me personally it was very important to get that I think, because then I could say that, okay I got this, I can do that, too and don't only get it from Formas, I got it from this basic research as well (...) And I think especially being an interdisci- 
plinary department I think that is important to show that I can attract that funding too, because that was the explanation why I didn't get [tenure] earlier, you have to show that you can attract money from VR." (Full professor)

Both the category of the "slacker" and the two ideal types of the "reliable" and "politically correct" researcher have become possible due to the increasing importance of grants in Swedish academia, and thus can be considered products of recent systemic changes. At the same time, they also illustrate distinct forms of existing in this system that reinforce a clear hierarchy of value assigned to disciplinary vs. interdisciplinary work. The "reliable academic" - being able to succeed in the competition for funds by the Swedish research council and similar types of offerings - is the only type that will easily satisfy both grant- and publishing-related tenure requirements. The "politically correct researcher", by contrast, draws on a limited set of issue-oriented funding sources. Although these have played an important historical role in establishing the center, they are widely considered less prestigious as well as subject to shifting trends. Together with "slackers" or "freeloaders," "politically correct researchers" are more likely to rely on the strategies of pooling and the use of portfolios, so as to bridge gaps between successful grant applications. A number of such academic biographies consisting of short-term contracts patched together through grant applications and makeshift budgetary arrangements - can be found among the staff of the center. A paradoxical effect of these solidary strategies thus is that they promote a differentiation of two divergent career paths. One career path is reserved for scholars who may have conducted doctoral or postdoctoral work at the center, but have successfully cultivated a research profile compatible with mono-disciplinary quality standards. Such an academic pedigree comes with greater career security through tenure and professorships. The other career path is marked by the possibility of pursuing research agendas outside disciplinary frameworks, but also by much fewer long-term career perspectives. In short, reliance on pooling and portfolios does allow researchers with a pronounced interdisciplinary focus to survive in academia, but it does go along with a social and epistemic marginalization compared to those who opt for being a "reliable academic."

\section{Discussion and Conclusions}

In this paper we have investigated how mid-career researchers in a Swedish research center at the intersection of natural and social sciences try to balance their interdisciplinary ambitions with the institutional career structure and funding conditions that underpin their work. Our analysis resonates in several ways with Fochler and Sigl's (2018) recent argument about "anticipatory uncertainty." Fochler and Sigl suggest that increasing competition for (project-based) funding, the spread of short-term contracts, and the rise of formal evaluation systems have created a de-facto governance of academic work that hampers the production of intellectually interesting and societally relevant research. Epistemic uncertainties and social uncertainties have become intertwined in such a way that researchers are incentivized to limit their pursuit of research that is too risky or unpredictable in terms of the accountable output 
(preliminary results, publications) it is likely to yield. Our own study has sought to disentangle the governance effects for interdisciplinary research that emerge from the interaction of policy incentives and formal requirements for advancing to more stable professional positions in the Swedish university system.

It is well known that interdisciplinary work poses specific practical and intellectual challenges due to greater preparatory effort and a lack of agreed-upon methodological and conceptual frameworks (Rhoten \& Pfirman 2007; Leahey 2007). Interestingly, the dedicated grant opportunities Swedish funders have created to stimulate policy-relevant research was instrumental in allowing the center to create an institutional space where researchers could productively embrace these challenges, at least temporarily. The strategy has been so successful that the center has come to play an important role in shaping the wider public debate on these questions. At the same time, while the affiliated junior and senior researchers care a great deal about interdisciplinary work, the need for them to work towards output that can be easily recognized as valid scholarly contributions by future employers and referees systematically undermines their interdisciplinary ambitions. Of particular analytical interest to us have been the work-arounds and makeshift solutions that researchers rely on to cope with such contradictory incentives. As concrete examples, we have discussed the use of collective resources buffers, portfolios, and 'multi-purpose' applications - these can be seen as attempts to maneuver a setting where different kinds of funding associated with diverse policy goals are available. One might thus say that while interdisciplinary work poses specific challenges for career development, it also creates special opportunities for dealing with them.

We have also gone a step further, however, in analyzing how techniques of exploiting such flexibility affect the situation of particular researchers who take part in these practices. As Fochler and Sigl (2018) have suggested, the practice of managing uncertainty through a portfolio-based organization of research (e.g., balancing scientifically risky projects with less ambitious but more predictable work) is an imperfect adaptation strategy, since it unevenly redistributes risks across the different levels of the academic career system (see also Müller 2012). In our own case, junior researchers who fail to submit successful grant applications or advance to tenured positions can only remain in the center on the condition that more successful colleagues are comfortable sharing the grant money and reputation they have acquired. It is through this tension between an originally collective research interest and individualized competition that the interdisciplinary ambitions of the center are effectively thwarted. Specifically, we have outlined how the success of individual researchers in attracting specific types of funding brings into being a finely stratified social hierarchy in the center - a distinction between "reliable researchers," "politically correct" ones, and the residual category of the "slacker." These categories can be seen as variants of what Ylijoki \& Henriksson (2017: 1294) have called academic career stories, i.e., shared ways of interpreting "what an academic career means and how it should be pursued." Career stories are both a product of recent systemic changes in university systems - in this case, the increasing importance of grants in Swedish academia - and also actively mediate how researchers make sense of these changes. Ylijoki \& Henriksson (2017) primarily distinguish between members of disciplinary "tribes" and a contractually precarious academic proletariat. Our own 
findings broaden this picture by showing how career stories enact and reinforce a value hierarchy of disciplinary vs. interdisciplinary work, even in a dedicated interdisciplinary institution.

This insight also qualifies a recent argument David Stark (2011) has made for the advantages of a broad set of heterarchical registers of value in organizations. In his analysis, the optimal conditions for commercial but also intellectual innovation apply whenever actors have the possibility to switch between registers in a flexible manner, thereby giving them the possibility to construct and exploit opportunities that would not emerge in a singular hierarchy of value (Rushforth et al. 2018). In our own case, the use of resource buffers, portfolios, and multi-purpose applications can be seen as a way of flexibly tapping into a variety of funding possibilities, which in principle are associated with incommensurable registers of worth, such as different forms of societal relevance and the ideal of academic excellence. However, the effectiveness of the work-arounds is undermined by the overriding normative power of formal career incentives, which continue to emphasize the ideal of the individual high-performing academic who publishes in disciplinary journals and is able to succeed in attracting the most selective grants. Under such circumstances, the workarounds themselves become an insidious mechanism that allows researchers to stay in academia, but systematically marginalizes their voices and epistemic ambitions in the process. Interdisciplinary work on ecological policy issues thereby becomes a possibility, but one that always represents the less prestigious counterpart to disciplinary research.

What are the broader implications of our analysis for science governance? A key implication is that interdisciplinary funding programs must be accompanied by interdisciplinary career policies. To be sure, arguments for more dedicated interdisciplinary policies have been made before. Felt et al. (2016), for example, have called for extending the funding periods of transdisciplinary work so as to prevent researchers from being forced to return to their "home fields" too quickly, and Hackett \& Rhoten (2009) have proposed possibilities in which interdisciplinary graduate education might be organized more effectively. While we fully support particularistic recommendations of this sort, we especially wish to emphasize the need for a more holistic view of science governance that would need to go along with such measures. Of crucial importance is not only ensuring that individual researchers are formally judged against suitable interdisciplinary standards, but that such appreciation for the specificities of interdisciplinarity also extends to the many informal contexts of assessment that pervade daily life in institutions (Rushforth \& de Rijcke 2015; Stark 2011). Our empirical material contains numerous mundane instances in which interdisciplinary researchers are judged by influential institutional actors (such as deans and colleagues in other departments), but outside the framework of formal evaluative routines. Such practices foster a sense among researchers that formal proclamations and commitments to interdisciplinarity are not much more than symbolic measures - the idea inevitably emerges that one may be "politically correct" but not actually a serious academic who is appreciated by the institution in the long term. In the absence of a comprehensive policy approach, researchers will continue to subordinate a principal heterarchy of funding opportunities to the onedimensional notion of 'success' that underpins the Swedish and many other career 
systems. The likely result of this is that interdisciplinary research will fail to unfold its full innovative potential in an academic landscape that is still characterized by thematic specialization.

Acknowledgments We would like to thank Maximilian Fochler for his valuable feedback on an earlier version of this article, as well as the two anonymous reviewers and the editors of Minerva for their thoughtful comments. Furthermore, we would like to thank Merle Jacob for her assistance in setting up the study.

Open Access This article is distributed under the terms of the Creative Commons Attribution 4.0 International License (http://creativecommons.org/licenses/by/4.0/), which permits unrestricted use, distribution, and reproduction in any medium, provided you give appropriate credit to the original author(s) and the source, provide a link to the Creative Commons license, and indicate if changes were made.

\section{References}

Allmendinger, Jutta. 2015. Quests for interdisciplinarity: A challenge for the ERA and HORIZON 2020 Policy Brief by the Research, Innovation, and Science Policy Experts (RISE). Luxembourg: Publications Office of the European Union. https://ec.europa.eu/research/openvision/pdf/rise/allmending er-interdisciplinarity.pdf. Accessed 11 Nov 2017.

Barry, Andrew, Georgina Born, and Gisa Weszkalnys. 2008. Logics of interdisciplinarity. Economy and Society 37(1): 20-49.

Boden, Margaret A. 1999. What is interdisciplinarity? In Interdisciplinarity and the organization of knowledge in Europe, ed. R. Cunningham, 13-24. Luxembourg: Office for Official Publications of the European Communities.

Boix Mansilla, Veronica. 2006. Symptoms of quality. Assessing expert interdisciplinary work at the frontier: an empirical exploration. Research Evaluation 15(1): 17-29.

Boix Mansilla, Veronica, Michèle Lamont, and Kyoko Sato. 2016. Shared Cognitive-Emotional-Interactional Platforms: Markers and Conditions for Successful Interdisciplinary Collaborations. Science, Technology, \& Human Values 41(4): 571-612.

Campbell, Lisa M. 2005. Overcoming obstacles to interdisciplinary research. Conservation Biology 19(2): 574-577.

Charmaz, Kathleen C. 2006. Constructing Grounded Theory. A Practical Guide for Qualitative Analysis. London: Sage.

Choucri, Nazli, Olivier de Weck, and Fred Moavenzadeh. 2006. Editorial: promotion and tenure for interdisciplinary junior faculty. MIT Faculty Newsletter (January/February). http://web.mit.edu/fnl/ volume/183/editorial.html. Accessed 11 Nov 2017.

Felt, Ulrike, Julia Igelsböck, Andrea Schikowitz, and Thomas Völker. 2013. Growing into what? The (un-)disciplined socialisation of early stage researchers in transdisciplinary research. Higher Education 65(4): 511-524.

Felt, Ulrike, Julia Igelsböck, Andrea Schikowitz, and Thomas Völker. 2016. Transdisciplinary Sustainability Research in Practice: Between Imaginaries of Collective Experimentation and Entrenched Academic Value Orders. Science, Technology, \& Human Values 41(4): 732-761.

Fisher, Donald, Janet Atkinson-Grosjean, and Dawn House. 2001. Changes in Academy/Industry/Staterelations in Canada: The Creation and Development of the Networks of Centres of Excellence. Minerva 39(3): 299-325.

Fochler, Maximilian, Ulrike Felt, and Ruth Müller. 2016. Unsustainable Growth, Hyper-Competition, and Worth in Life Science Research: Narrowing Evaluative Repertoires in Doctoral and Postdoctoral Scientists' Work and Lives. Minerva 54(2): 175-200.

Fochler, Maximilian, and Lisa Sigl. 2018. Anticipatory Uncertainty: How Academic and Industry Researchers in the Life Sciences Experience and Manage the Uncertainties of the Research Process Differently. Science as Culture 27(3): 349-374. 
Funtowicz, Silvio O., and Jerome R. Ravetz. 1993. The emergence of post-normal science. In Science, politics, and morality: Scientific uncertainty and decision making, ed. R. von Schomberg, 85-123. Dordrecht: Kluwer.

Gläser, Jochen, Stefan Lange, Grit Laudel, and Uwe Schimank. 2010. Informed Authority? The Limited Use of Research Evaluation Systems for Managerial Control in Universities. In Reconfiguring Knowledge Production: Changing Authority Relationships in the Sciences and Their Consequences for Intellectual Innovation, eds. Richard Whitley, Jochen Gläser, and Lars Engwall, 149-183. Oxford: Oxford University Press.

Hackett, Edward J. 1987. Funding and Academic Research in the Life Sciences: Results of an Exploratory Study. Science \& Technology Studies 5(3): 134-147.

Hackett, Edward J., and Diana R. Rhoten. 2009. The Snowbird Charrette: Integrative Interdisciplinary Collaboration in Environmental Research Design. Minerva 47(4): 407-440.

Håkansta, Carin, and Merle Jacob. 2016. Mode 2 and the Tension Between Excellence and Utility: The Case of a Policy-Relevant Research Field in Sweden. Minerva 54(1): 1-20.

Hamann, Julian. 2016. The visible hand of research performance assessment. Higher Education 72(6): 761-779.

Hammarfelt, Björn, and Sarah de Rijcke. 2015. Accountability in Context: Effects of Research Evaluation Systems on Publication Practices, Disciplinary Norms, and Individual Working Routines in the Faculty of Arts at Uppsala University. Research Evaluation 24(1): 63-77.

Hicks, Diana. 2012. Performance-Based University Research Funding Systems. Research Policy 41(2): 251-261.

Jacob, Merle. 2015. RIO Country Report Sweden 2014. http://doi.org/10.2791/103569

Klein, Julie T. 2000. A conceptual vocabulary of interdisciplinary science. In Practising Interdisciplinarity, eds. Peter Weingart and Nico Stehr, 3-24. Toronto: University of Toronto Press.

Klein, Julie T. 2010. A taxonomy of interdisciplinarity. In The Oxford Handbook of Interdisciplinarity, eds. R. Frodeman, J.T. Klein, and C. Mitcham, 15-30. Oxford: Oxford University Press.

Knorr-Cetina, Karin. 1981. The manufacture of knowledge: An essay on the constructivist and contextual nature of science. Oxford, New York: Pergamon Press.

Lattuca, Lisa R. 2001. Creating interdisciplinarity: Interdisciplinary research and teaching among college and university faculty. Nashville, TN: Vanderbilt University Press.

Laudel, Grit. 2006. The art of getting funded: How scientists adapt to their funding conditions. Science and Public Policy 33(7): 489-504.

Leahey, Erin. 2007. Not by productivity alone: How visibility and specialization contribution to academic earnings. American Sociological Review 72(4): 533-561.

Leisyte, Liudvika. 2007. University Governance and Academic Research - Case Studies of Research Units in Dutch and English Universities. Doctoral Dissertation, University of Twente, Enschede. Unpublished.

Lindvig, Katrine, and Line Hillersdal. 2019. Strategically Unclear? Organising Interdisciplinarity in an Excellence Programme of Interdisciplinary Research in Denmark. Minerva 57(1): 23-46.

Müller, Ruth. 2012. Collaborating in Life Science Research Groups: The Question of Authorship. Higher Education Policy 25(3): 289-311.

Müller, Ruth. 2014a. Postdoctoral Life Scientists and Supervision Work in the Contemporary University: A Case Study of Changes in the Cultural Norms of Science. Minerva 52(3): 329-349.

Müller, Ruth. 2014b. Racing for What? Anticipation and Acceleration in the Work and Career Practices of Academic Life Science Postdocs. Forum Qualitative Sozialforschung / Forum: Qualitative Social Research 15(3). http://nbn-resolving.de/urn:nbn:de:0114-fqs1403150

National Academies. 2005. Facilitating interdisciplinary research. Washington, DC: National Academies Press. https://www.nap.edu/catalog/11153/facilitating-interdisciplinary-research. Accessed 11 Nov 2017.

Nowotny, Helga, Peter Scott, and Michael Gibbons. 2001. Re-thinking science: Knowledge and the public in an age of uncertainty. Cambridge: Polity Press.

Parker, John N., and Edward J. Hackett. 2012. Hot Spots and Hot Moments in Scientific Collaborations and Social Movements. American Sociological Review 77(1): 21-44.

Peterson, David. 2017. The depth of fields: Managing focus in the epistemic subcultures of mind and brain science. Social Studies of Science 47(1): 53-74.

Pfirman, Stephanie, and Paula J.S. Martin. 2010. Facilitating interdisciplinary scholars. In The Oxford Handbook of Interdisciplinarity, eds. R. Frodeman, J.T. Klein, and C. Mitcham, 387-403. Oxford: Oxford University Press. 
Porter, Alan L., Alex S. Cohen, J. David Roessner, and Marty Perreaul. 2007. Measuring researcher interdisciplinarity. Scientometrics 72(1): 117-147.

Rafols, Ismael, Loet Leydesdorf, Alice O'Hare, Paul Nightingale, and Andy Stirling. 2012. How journal rankings can suppress interdisciplinary research: A comparison between Innovation Studies and Business \& Management. Research Policy 41(7): 1262-1282.

Rhoten, Diana R., and Stephanie Pfirman. 2007. Women in interdisciplinary science: Exploring preferences and consequences. Research Policy 36(1): 56-75.

Rinia, Ed J., Thed van Leeuwen, Eppo W. Bruins, Hendrik G. van Vuren, and Anthony F.J. van Raan. 2001. Citation delay in interdisciplinary knowledge exchange. Scientometrics 51(1): 293-309

Rittel, Horst W., and Melvin Webber. 1973. Dilemmas in a General Theory of Planning. Policy Sciences 4(2): 155-169.

Rushforth, Alexander, and Sarah de Rijcke. 2015. Accounting for Impact? The Journal Impact Factor and the Making of Biomedical Research in the Netherlands. Minerva 53(2): 117-139.

Rushforth, Alexander, Thomas Franssen, and Sarah de Rijcke. 2018. Portfolios of Worth: Capitalizing on Basic and Clinical Problems in Biomedical Research Groups. Science, Technology, \& Human Values. https://doi.org/10.1177/0162243918786431.

Sarewitz, Daniel. 2010. Against Holism. In The Oxford Handbook of Interdisciplinarity, eds. R. Frodeman, J.T. Klein, and C. Mitcham, 65-75. Oxford: Oxford University Press.

Science Europe. 2012. Science Europe Position Statement. Horizon 2020: Excellence Counts. http:// www.scienceeurope.org/wp-content/uploads/2014/05/SE_H2020_Excellence_Counts_FIN.pdf.

Sigl, Lisa. 2015. On the Tacit Governance of Research by Uncertainty: How Early Stage Researchers Contribute to the Governance of Life Science Research. Science, Technology, \& Human Values 41(3): 347-374.

Sivertsen, Gunnar. 2008. Experiences with a Bibliometric Model for Performance Based Funding of Research Institutions. In Book of Abstracts, 10th International Science and Technology Indicators Conference, eds. J. Goriaz and E. Schiebel, 126-8, 17-20 September, Austria: University of Vienna.

Spanner, Don. 2001. Border crossings: understanding the cultural and informational dilemmas of interdisciplinary scholars. Journal of Academic Librarianship 27(5): 352-360.

Star, Susan L. 1985. Scientific work and uncertainty. Social Studies of Science 15(3): 391-427.

Stark, David C. 2011. The Sense of Dissonance: Accounts of Worth in Economic Life. Princeton: Princeton University Press.

Strathern, Marilyn. 2004. Commons and Borderlands: Working Papers on Interdisciplinarity, Accountability and the Flow of Knowledge. Wantage: Sean Kingston Publishing

Strathern, Marilyn. 2007. Interdisciplinarity: Some models from the human sciences. Interdisciplinary Science Reviews 32(2): 123-134.

Strauss, Anselm, and J. Corbin. 1998. Basics of Qualitative Research Techniques and Procedures for Developing Grounded Theory. Thousand Oaks, CA: Sage.

Wald, Andreas E. 2007. The Effect of 'Mode 2'-Related Policy on the Research Process: The Case of Publicly Funded German Nanotechnology. Science \& Technology Studies 20(1).

Weingart, Peter, and Nico Stehr (eds.). 2000. Practising Interdisciplinarity. London: University of Toronto Press.

Whitley, Richard. 2007. The Consequences of Establishing Research Evaluation Systems for Knowledge Production in Different Countries and Scientific Fields. In The Changing Governance of the Sciences. Sociology of the Sciences Yearbook, eds. R. Whitley and J. Gläser, 3-27. Dordrecht: Springer.

Whitley, Richard. 2010. Reconfiguring the public sciences: The impact of governance changes on authority and innovation in public science systems. In Reconfiguring Knowledge Production: Changing Authority Relationships in the Sciences and Their Consequences for Intellectual Innovation, eds. R. Whitley, J. Gläser, and L. Engwall, 3-47. Oxford: Oxford University Press.

Ylijoki, Oili-Helena, and Lea Henriksson. 2017. Tribal, proletarian and entrepreneurial career stories: Junior academics as a case in point. Studies in Higher Education 42(7): 1292-1308.

Publisher's Note Springer Nature remains neutral with regard to jurisdictional claims in published maps and institutional affiliations. 